Original Article

\title{
Causative Organisms and their Sensitivity Patterns in Urinary Tract Infection in Children
}

\author{
Deve Das, Muhammad Nadeem Chohan, Srichand Talreja
}

\begin{abstract}
OBJECTIVE: To determine causative organisms and their sensitivity patterns in urinary tract infection in children, attending Pediatric Department Dow University Karachi.

METHODOLOGY: This Cross sectional study was conducted at pediatric Department, Civil Hospital Karachi from July to December 2014. A total of 150 children between age 1 month to 15 years having the probable Urinary Tract Infection, attending the Pediatric OPD or admitted to ward were enrolled. Urine Culture and Sensitivity sample was collected aseptically. In infants and younger children's, it was collected by urethral catheterization and in older children collected by mid-stream clean catch void, after proper local cleaning, while in young infant collected by suprapubic bladder aspiration.

RESULTS: Among a total of 150 children, mean age of participants was $4.4 \pm 3.4$ years, $70.7 \%$ were above five years of age, $52 \%$ were males with male to female ratio were 1.1:1. Urine culture was positive in $\mathbf{2 9 . 3 \%}$ cases, among them $\mathbf{5 5 \%}$ were positive for E. coli, $18 \%$ for Pseudomonas, $16 \%$ for Proteus and $11 \%$ for Klebsiella. $\mathbf{7 0 . 8} \%$ E. coli were not sensitive to amoxicillin and ampicillin. $\mathbf{7 5 \%}$ pseudomonas was resistant to ampicillin and amoxicillin. There was no difference in in-vitro and in-vivo response of E. coli to ampicillin and amoxicillin.

CONCLUSION: It is concluded from this study that most common organism in Pediatric UTI was E. coli especially in 1-5 years' age group and most of it were resistant to amoxicillin and ampicillin.
\end{abstract}

KEYWORDS: Organism, Antibiotic sensitivity, Children.

This article may be cited as: Das D, Chohan MN, Talreja S. Causative Organisms and their Sensitivity Patterns in Urinary Tract Infection in Children. J Liaquat Uni Med Health Sci. 2019;18 (02):119-24. doi: 10.22442/jlumhs.191820613

\section{INTRODUCTION}

Urinary tract infection (UTI) cause significant morbidity in children. ${ }^{1}$ Prevalence of UTI in children is $3-5 \%$, prevalence is different in various age groups and sex of child. Male: female ratio is 2.8 to $5.4: 1$ in first year of life, but it significantly increased in females with male: female ratio of $1: 10$ after 1 year of age. Prevalence of UTI is 3-7\% in girls and $1-2 \%$ in boys ${ }^{15}$. Prevalence rate is $2.1 \%$ in males and $2.4 \%$ in females ${ }^{16}$.

First episode of UTI in female usually occurs by the age of 5 years, with peaks during toilet training and infancy. Within 18 months of first UTI, 60 to $80 \%$ of females will develop second episode of UTI ${ }^{2,3}$.

UTI is more common in uncircumcised boys. Organisms responsible for UTI are variable in children and particularly depend upon factors such as age and geographic location of patient ${ }^{4}$. Escherichia Coli is reported as the most common organism (43.3-71\%) so far followed by Klebsiella (13-30\%), Pseudomonas $(16.66 \%)$ and Proteus $(11 \%)^{4,5}$. Escherichia Coli, Klebsiella and Proteus are responsible for 75 to $90 \%$ of all infection in females. Some reports suggest
Proteus is more common as $\mathrm{E}$. Coil in males during $1^{\text {st }}$ year of age while other reports indicate grams' positive organisms are more prevalent. ${ }^{2}$ Gold standard test for the diagnosis of UTI is Urine Culture ${ }^{2,6}$.

Although standard treatment of acute cystitisincludes cotrimoxazole, Nitrofurantoin and amoxicillin while ceftriaxone, ampicillin, gentamicin, and Cefixime are used for pyelonephritis ${ }^{2,4}$. As organism causing UTI vary in different geographical areas, so aim of this study is to know the causative organism of UTI and their sensitivity and resistance patterns in our locality to recommend antimicrobials use in accordance with the results.

\section{METHODOLOGY}

A Cross sectional study done by Non-probability consecutive sampling in 150 children, from July to December 2014 at Department of Pediatrics Dow University Karachi. Approval was taken from the Ethical review committee of institute. All patients visiting Pediatric Out Door Department fulfilling inclusion criteria: Children aged one month to fifteen year of age with either sex with clinical features suggestive of UTI such as, Foul-smelling urine, 
abdominal pain, Irritability, Vomiting, Enuresis, dysuria, urgency, frequency, Fever, Flank/back pain, Incontinence, poor feeding, were advised for Urine Analysis. Children whose Urine analysis report showed Pus Cell > 10 along with Presence of Nitrates were enrolled in the study. Written and Verbal consent was taken from the parents. Sample size calculated by using the open Epi software, taking 95\% confidence interval, prevalence $15 \%$ error at $5 \%$.

Children having recurrent UTI, congenital renal anomalies, Nephritic/Nephrotic syndrome/Chronic renal failure, Malaria, Typhoid, Sepsis, and Meningitis were excluded from the study. Patients who had already received antibiotics and those having perianal rash were also excluded from the study.

For urine C/S, sample was collected aseptically (in infants and younger children it was collected by urethral catheterization and in older children collected by clean mid-stream void after proper local cleaning while in young infant it was collected by suprapubic bladder aspiration) in sterile container and were sent to pathology laboratory of Civil Hospital Karachi.

Specimens were cultured on cystine-, lactose- and electrolyte-deficient (CLED) agar plates followed by incubation under aerobic conditions at $37^{\circ} \mathrm{C}$ for 24 hours. Those bacterial cultures containing bacterial growth of $>10^{5}$ colony forming unit (CFU)/ ml were considered significant, while CFU count of less than $10^{5}$ were considered non-significant for the analysis of bacteriuria. Bacterial colonies were identified by their morphological characteristics and biochemical characterisation through gram's staining, catalase test and bile aesculin hydrolysis test. Antimicrobial susceptibility profiling was done by modified Kirby Bauer disc-diffusion method. Bacterial suspensions having turbidity comparable to that of $0.5 \mathrm{McF}$ arland standard were applied on Mueller-Hinton agar plates. Antibiotic discs of analytical grade (Oxoid, UK) used as follows: ampicillin $(10 \mu \mathrm{g})$, amoxicillin $(10 \mu \mathrm{g})$, gentamicin $(10 \mu \mathrm{g})$, ciprofloxacin $(5 \mu \mathrm{g})$, nitrofurantoin $(300 \mu \mathrm{g})$, fosfomycin $(200 \mu \mathrm{g})$. Sensitivity of bacterial strains was assessed by measuring the zones of inhibition in millimeters following the Clinical and Laboratory Standards Institute guidelines. American type culture collection (ATCC® 25923) strain of Staphylococcus aureus was used as quality control standard. Positive Urine Cultures were further tested for their sensitivity and resistance to various antibiotics.

Data was entered and analyzed in SPSS version 22.0. Quantitative variables like age and duration of UTI were calculated by using Descriptive statistics i.e. mean+standard deviation. Qualitative variables like gender, type of bacteria, drugs resistance and sensitivity were calculated by Frequency and percentage. Stratification was done with regard to age, gender and duration of UTI to see effect of this on outcome.

\section{Operational Definitions}

Proven Urinary tract infection: Defined as presence of single organism colony count $>10^{5} \mathrm{CFU} / \mathrm{ml}$ in freshly voided mid- stream urine sample and urethral catheterization or any number of single organism in urine sample collected through supra -public puncture method.

Probable Urinary Tract Infection: Having features of UTI (Fever, Abdominal Pain, Dysuria, Urinary Frequency) and the Presence of $>10$ Pus Cell and Nitrates in Urine.

Susceptibility testing: It was performed in isolated microorganisms that are responsible for infectious process requiring antimicrobial therapy. On the basis of these testing bacteria were divided into categories "Sensitive", "Intermediate" and "Resistant".

\section{RESULTS}

A total of 150 children were enrolled during study period. The mean age was $4.4 \pm 3.4$ years (Table II), $44(70.7 \%)$ were $\geq$ five years of age, 78 $(52 \%)$ were males with male to female ratio 1.1:1 (Table II). Duration of UTI is summarized in table 2. Urine culture was positive in $44(29.3 \%)$ cases (Table I). Most common clinical presentation was Fever that was present in 58 (38.66) children (Table III). The most commonly isolated organism was $\mathrm{E}$. coli in 24 $(55 \%)$ cases followed by Pseudomonas 8 (18\%). $70.8 \%$ E. coli were resistant to both ampicillin and amoxicillin (Graph I). $75 \%$ of pseudomonas was resistant to ampicillin and amoxicillin (Graph II). There was no difference in in-vitro and in-vivo response of $\mathrm{E}$. coli to ampicillin and amoxicillin response. Antimicrobial sensitivity pattern of Proteus and Klebsiella were summarized in Graph III and IV).

TABLE I: STRATIFICATION OF URINE CULTURE POSITIVE CASE BY AGE $(n=150)$

\begin{tabular}{|l|c|c|c|c|c|}
\hline \multirow{2}{*}{ Urine culture } & \multicolumn{4}{|c|}{ Age Group* } & \multirow{2}{*}{ Total } \\
\cline { 2 - 5 } & $<1$ year & $\mathbf{1 - 5}$ Years & $\mathbf{6 - 1 0}$ years & $>10$ years & \\
\hline Positive & $2(4.5 \%)$ & $25(54.5 \%)$ & $12(27.3 \%)$ & $6(13.6 \%)$ & $\mathbf{4 4}$ \\
\hline Negative & $16(15.1 \%)$ & $64(60.4 \%)$ & $20(18.9 \%)$ & $6(5.7 \%)$ & $\mathbf{1 0 6}$ \\
\hline Total & $\mathbf{1 8}$ & $\mathbf{8 8}$ & $\mathbf{3 2}$ & $\mathbf{1 2}$ & $\mathbf{1 5 0}$ \\
\hline
\end{tabular}


Deve Das, Muhammad Nadeem Chohan, Srichand Talreja

TABLE II:

DEMOGRAPHY OF STUDY PARTICIPANTS $(n=150)$

\begin{tabular}{|l|c|c|}
\hline \multicolumn{1}{|c|}{ Age group } & Frequency & Percentage \\
\hline$<1$ year & 18 & 12.0 \\
\hline $1-5$ years & 88 & 58.7 \\
\hline $6-10$ Years & 32 & 21.3 \\
\hline$>10$ years & 12 & 8.0 \\
\hline Male & 78 & 52.0 \\
\hline Female & 72 & 48 \\
\hline Duration of UTI $\leq 5$ days & 83 & 55.3 \\
\hline Duration of UTI $>5$ days & 67 & 44.7 \\
\hline Urine Culture (Positive) & $\begin{array}{c}44 \\
\text { Males 24(54.5\%) } \\
\text { Females 20(45.5\%) }\end{array}$ & 29.3 \\
\hline Urine Culture (No growth) & 106 & 70.7 \\
\hline
\end{tabular}

TABLE III: CLINICAL MANIFESTATION $(n=150)$

\begin{tabular}{|l|c|c|}
\hline \multicolumn{1}{|c|}{ Complaints } & Frequency & Percentage \\
\hline Fever & 58 & 38.66 \\
\hline Abdominal Pain & 34 & 22.66 \\
\hline Dysuria & 25 & 16.66 \\
\hline Enuresis & 6 & 4.00 \\
\hline Vomiting & 9 & 6.00 \\
\hline Urinary Urgency & 18 & 12.00 \\
\hline
\end{tabular}

sensitivity test should be performed in every case to know the sensitivity pattern of specific bacteria. After getting the Urine $\mathrm{C} / \mathrm{S}$ result antibiotic with narrowest spectrum and little adverse effect should be selected. In this study urine culture was positive in $29.3 \%$ cases. E. coli was most common organism isolated accounting for $55 \%$ of cases. Amongst E. coli $70.8 \%$ were resistant to ampicillin and amoxicillin, 54.2\% resistant to co-trimoxazole, $13-16.7 \%$ resistant to third generation cephalosporin, and $12.5 \%$ resistant to gentamicin. E. coli was sensitive to Fosphomycin and nitrofurantoin. Our study is compatible with a study done at Pakistan Institute of Medical Sciences, Islamabad in which $\mathrm{E}$. coli $(57.2 \%)$ was the commonest isolate, among them only $38.5 \% \mathrm{E}$ coli were sensitive to ampicillin ${ }^{18}$.

Similarly, in a study ampicillin and Cotrimoxazole had the high resistance rates, while nitrofurantoin had low resistance rates. Unlike this study coamoxiclav or third-generation cephalosporin had no bacterial resistance ${ }^{10}$.

In another local study, unlike to our study E. coli was present in $14.84 \%$, Klebsiella Pneumoniae, 10.32\%, Staphylococcus Saprophyticus $2.58 \%$ urine Culture but like our study these bacterial pathogens were sensitive to Amoxycillin-Clavulanic acid but unlike our study it was also sensitive to TrimethoprimSulfamethoxazole ${ }^{17}$.

In another similar local study, E. coli $(57.3 \%)$ was frequent organism, followed by Klebsiella (18.8\%), Staphylococcus aureus (13.7 \%), Pseudomonas $(5.1 \%)$ and Proteus $(3.4 \%)$. All isolates were sensitive to ciprofloxacin, amikacin, cefixime and imepenem.

\section{GRAPH I: SENSITIVITIES OF E. COLI $(n=24)$}

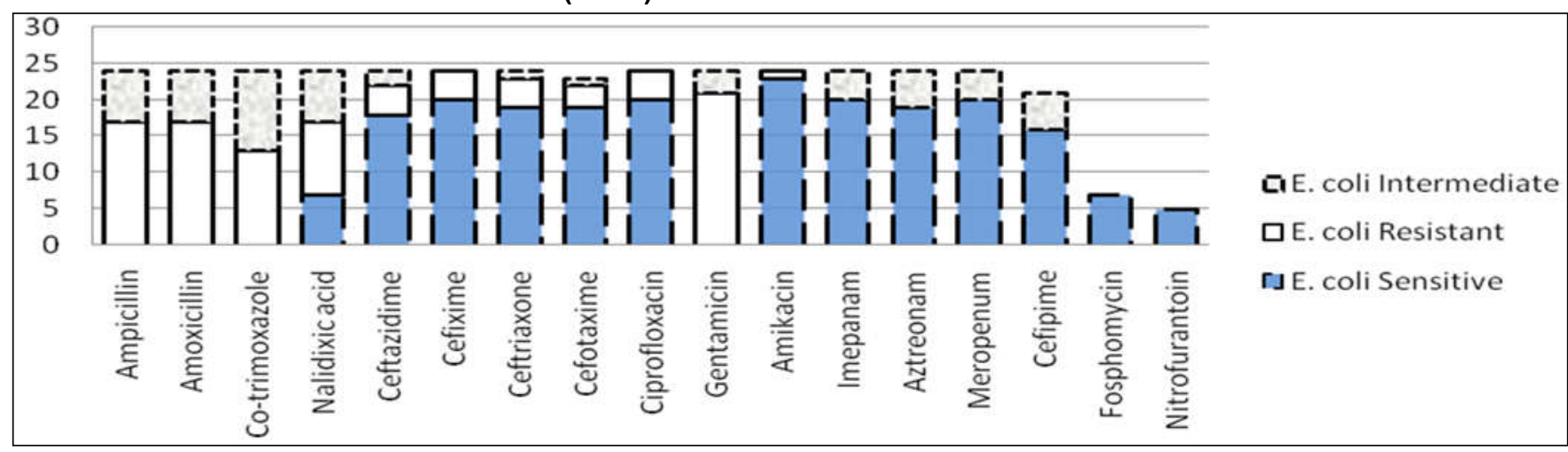

Note: Not tested (NT) for Cefotaxime (1), Cefipime (3), Fosphomycin (17) and Nitrofurantoin (19)

\section{DISCUSSION}

While selecting antibiotics, its resistance pattern is a big concern. Bacteria are getting resistant to antibiotics used for the treatment of UTI ${ }^{7}$. Studies showed the various resistance patterns of pathogens causing $\mathrm{UTI}^{8,9}$. While treating UTI; routine Urine culture and
Similarly, $38.5 \% \mathrm{E}$ coli and $37.5 \%$ Klebsiella were sensitive to Ampicillin, and $14.3 \% \mathrm{E}$ coli and $9.1 \%$ Klebsiella were sensitive to Trimethoprimsulphamethoxazole ${ }^{18}$.

In another similar local study, isolates were Escherichia coli $(50 \%)$, E. faecalis (22.5\%), 


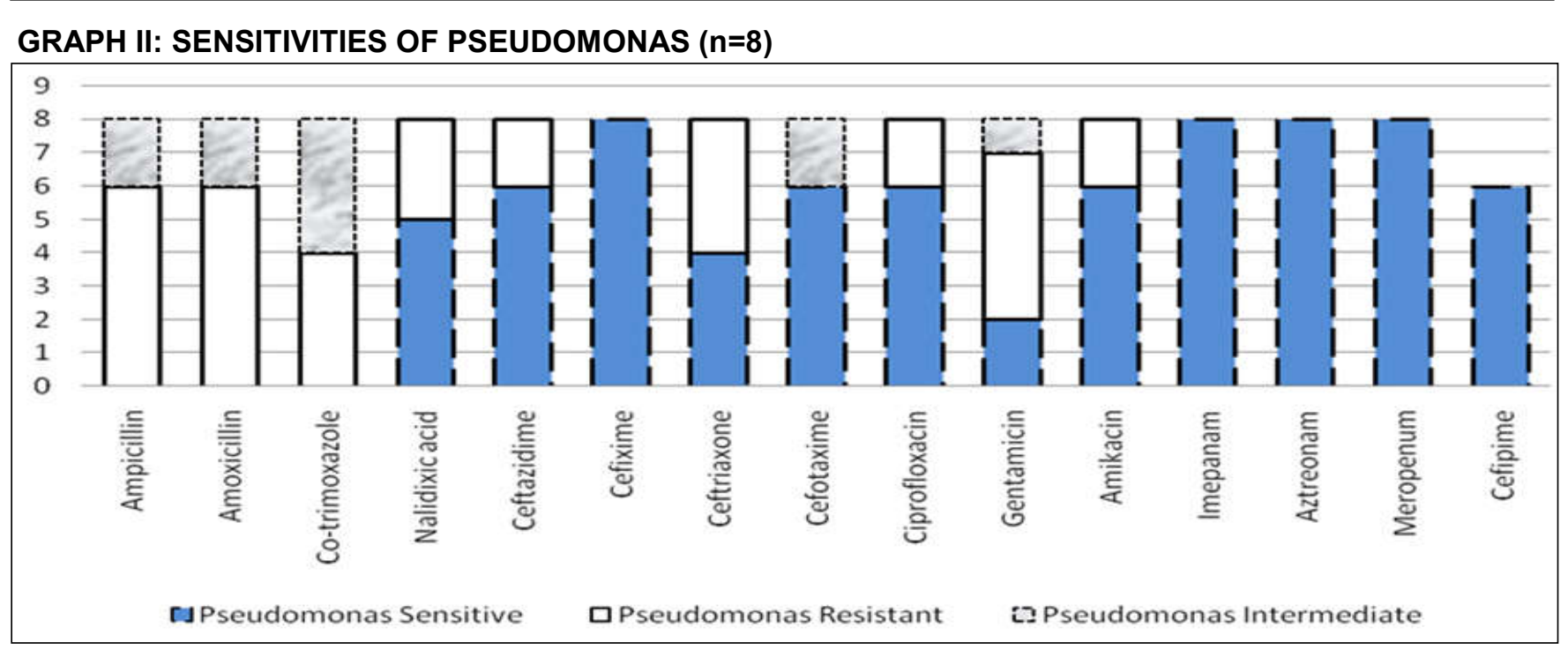

Note: Not tested (NT) for Cefipime (2), Fosphomycin (8) and Nitrofurantoin (8)

GRAPH III: SENSITIVITIES OF PROTEUS $(n=7)$

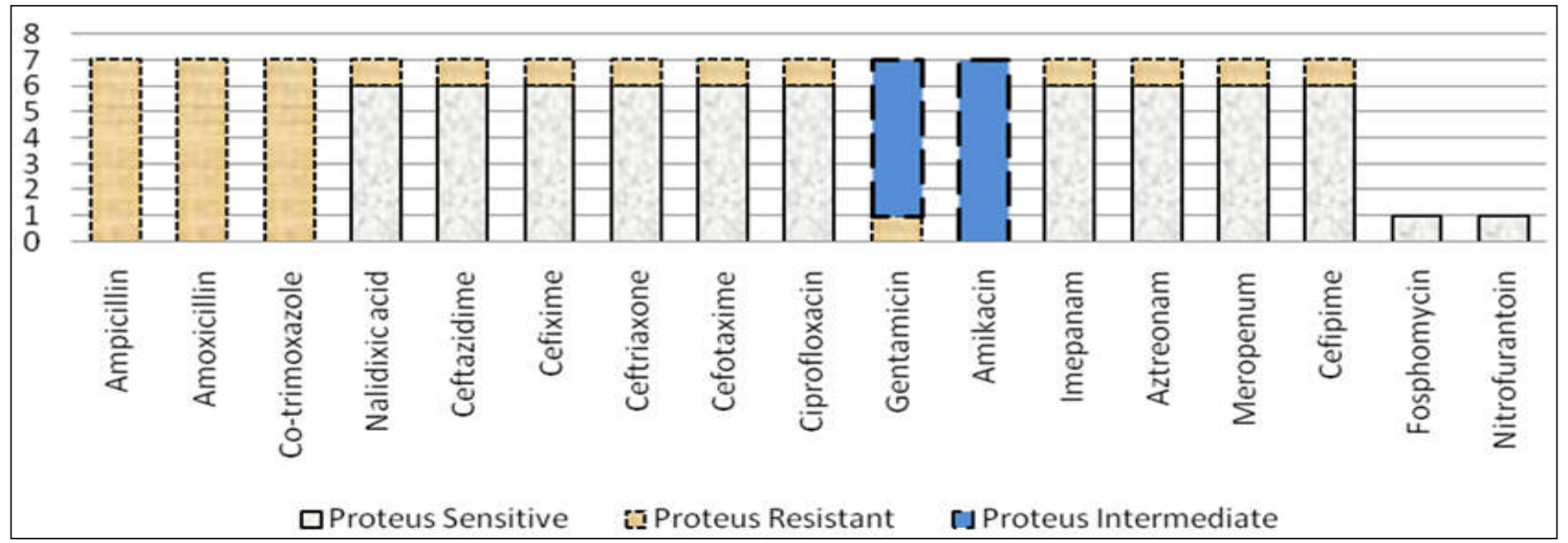

Note: Not tested (NT) for Fosphomycin (6) and Nitrofurantoin (6)

GRAPH IV: SENSITIVITIES OF KLEBSIELLA $(n=5)$

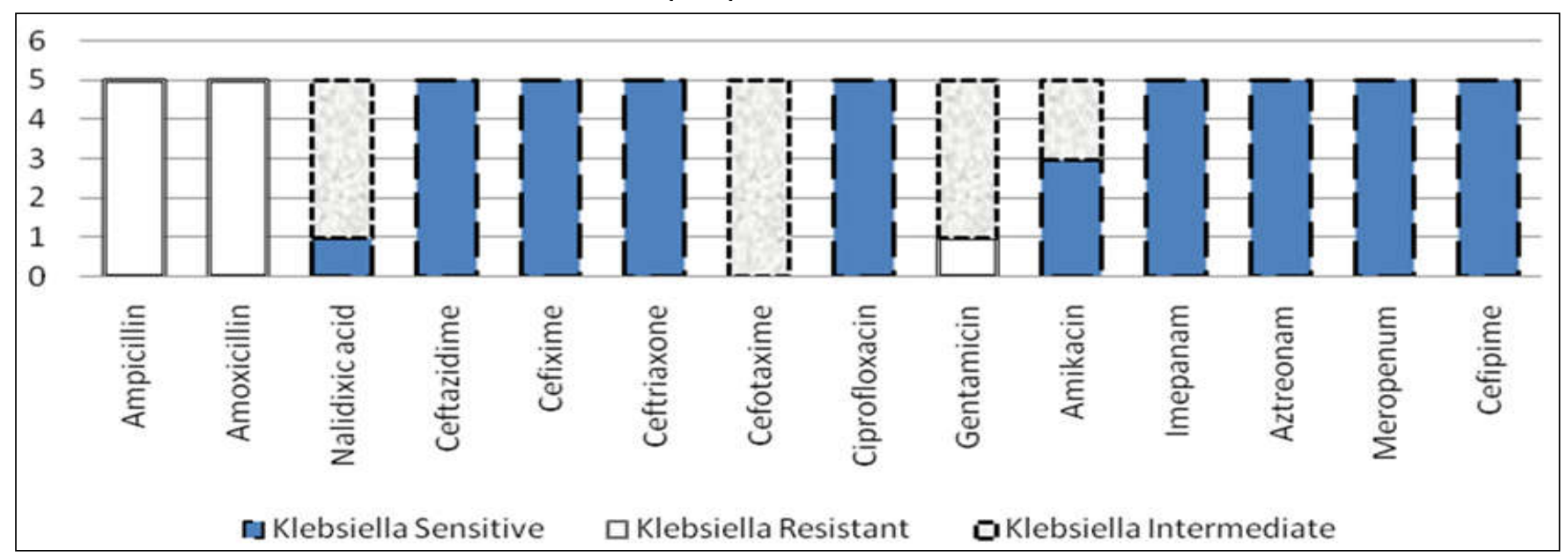

Note: Not tested (NT) for Fosphomycin (5) and Nitrofurantoin (5) 
P. aeruginosa $(12.5 \%)$, S. aureus $(10 \%)$ and Klebsiella (5\%). Importantly Amoxillin was resistance to almost all pathogens. Similarly, Fosfomycin and Linezolid showed $100 \%$ sensitivity pattern, in patients with E. coli and E. faecalis ${ }^{19}$.

In another different local study E-coli was found in 11 $(18.33 \%)$ males and $21(35 \%)$ females, citrobacter 2 $(3.33 \%)$ in males and $12(20 \%)$ in females, enterobactericae $9(15 \%)$ in males, proteus $1(1.66 \%)$ in males and $2(3.33 \%)$ in females, pseudomonas 2 $(3.33 \%)$ in males and $1(1.66 \%)$ in females, salmonella $1(1.66 \%)$ in males and none in females ${ }^{20}$.

In another similar international study, E. coli was $(54.80 \%)$ causing UTI. However, the resistant patterns were different from this study. Resistance rates of $\mathrm{E}$. coli isolates were $85.9 \%$ to co-trimoxazole, $80.0 \%$ to penicillin, $77.0 \%$ to ampicillin, $68.0 \%$ to chloramphenicol, $12.9 \%$ to ciprofloxacin, $12.9 \%$ to ceftriaxone, $12.9 \%$ tocephalothin, and $14.0 \%$ to amikacin $^{11}$. In another different study $E$. coli was the most common isolated organism accounted for $71 \%$ cases unlike this study the number is higher ${ }^{5}$.

Pseudomonas causes UTI in children; worldwide antibiotic susceptibility patterns are different in different countries ${ }^{12}$. In this study Pseudomonas was second common pathogen accounted for $18 \%$ cases. Pseudomonas was resistant to both ampicillin and amoxicillin, each accounted for $75 \%$ cases. This was also resistant to co-trimoxazole in $50 \%$ cases, nalidixic acid in $37.5 \%$ cases, and ceftazidime in $25 \%$ and ciprofloxacin in $25 \%$ cases. Similar study in Iran reported same resistant patters except all pathogens were sensitive to ciprofloxacin ${ }^{11}$.

In another similar local study most common uropathogens were E. coli $(72.7 \%)$ and Klebsiella pneumonia (8.3\%), among them E. coli was resistant to ampicillin in $63.3 \%$ of cases and to co-trimoxazole in $42.6 \%$ of cases $^{13}$. In another study $E$. coliwas not sensitive to ampicillin $(44,8 \%)$ and to first generation cephalosporin $(36 \%)$ while in our study resistant pattern is different ${ }^{14}$.

In another local study $\mathrm{E}$. coli was the predominant isolate $(165 / 225)$ followed by Klebsiella sp (30/225) and Pseudomonas (13/225). Similarly, all gram negative bacteria in general were found to be resistant to the commonly used antibiotics and were sensitive to amikacin, nitrofurantoin, imipenem, cefoperazone/ sulbactam and piperacillin/tazobactam ${ }^{21}$.

In another similar study isolated organism were $\mathrm{E}$. coli $(65.1 \%)$ followed by E. fecalis (20.8\%). Similarly, there was good sensitivity with Vancomycin, Amikacin, Nitrofurantoin and Impinime while there was high resistance with Ampicillin and Ceftriaxone ${ }^{22}$.

In another similar study the most common microorganism isolated was Escherichia coli 41
(71.9\%) followed by Klebsiella $7(12.3 \%)$, Proteus 5 (8.8\%), Pseudomonas 2(3.5\%), Staphylococcus aureus $1(1.8 \%)$ and Candida albicans $1(1.8 \%)$. The microorganisms were most resistant to Ampicillin 37 (90\%), Trimethoprim sulphamethoxazole 35(85.4\%). The most sensitive drugs were Gentacyn 35(85.4\%) and Nitrofurantoin $34(82.9 \%)^{23}$.

\section{CONCLUSION}

It is concluded from this study that most common organism in Pediatric UTI was E. coli especially in 1-5 years' age group and most of it were resistant to amoxicillin and ampicillin.

Ethical Permission: CPSP REU No. 5451 dated: 25-05-2015.

Conflict of Interest: All authors have no conflict of interest and none to declare.

Funding: None to declare

\section{REFERENCES}

1. Anis-ur-Rehman, Jahanzeb M, Siddique TS, Idris $M$. Frequency and clinical presentation of UTI among children of Hazara division of Pakistan. J Ayub Med Coll Abbottabad. 2008; 20(1): 63-5.

2. Elder JS. Kliegman RM, Behrman RE, Jenson $\mathrm{HB}$, Stanton, editors. Urinary Tract Infection, Nelson text book of pediatrics. Philadelphia: Saunders; 2008. p. 2223-8.

3. Muoneke VU, Ibekwe MU, Ibekwe RC. Childhood Urinary Tract Infection in Abakaliki: Etiological Organisms and Antibiotic Sensitivity Pattern. Ann Med Health Sci Res. 2012; 2(1):29-32. doi: 10.4103/2141-9248.96933.

4. Hafeez F, Anwar S, Ahmad TM. Urinary tract infection in children: Clinical and etiological pattern. Pak Pediatr J 2005; 29(1):35-40.

5. Qureshi AM. Organism causing urinary tract infection in pediatric patient at Ayub teaching hospital Abbottabad. J Ayub Med Coll Abbottabad. 2005; (1): 72-4.

6. Zorc JJ, Kiddoo DA, Shaw KN. Diagnosis and management of urinary tract infections. Clin Microbiol Rev. 2005; 18(2):417-22.

7. Gruneberg RN. Changes in urinary pathogens and their antibiotic sensitivities, 1971-1992. J Antimicrob Chemother. 1994; 33(suppl A): 1-8.

8. Barrett SP, Savage MA, Rebec MP, Guyot A, Andrews N, Shrimpton SB. Antibiotic sensitivity of bacteria associated with community-acquired urinary tract infection in Britain. J Antimicrob Chemother.1999; 44(3): 359-65.

9. Gupta K, Scholes D, Stamm WE. Increasing prevalence of antimicrobial resistance among uropathogens causing acute uncomplicated 
cystitis in women. JAMA 1999; 281(8):736-8.

10. Borsari AG, Bucher B, Brazzola P, Simonetti GD, Dolina M, Bianchetti MG. Susceptibility of Escherichia coli strains isolated from outpatient children with community-acquired urinary tract infection in southern Switzerland. Clin Ther. 2008; 30(11): 2090-5. doi: 10.1016/j.clinthera. 2008.11.002.

11. Kalantar E, Motlagh $M$, Lornejad $H$, Reshadmanesh N. Prevalence of urinary tract pathogens and antimicrobial susceptibility patterns in children at hospital in Iran. Iranian J Clin Inf Dis. 2008; 3(3): 149-53.

12. Lutter SA, Currie ML, Mitz LB, Greenbaum LA. Antibiotic resistance patterns in children hospitalized for urinary tract infections. Arch Pediatr Adolesc Med. 2005; 159(10): 924-8.

13. Echeverri C Velez, Serna-Higuita LM, Serrano AK, Ochoa-Garcia C, Rojas Roses L, Maria Bedoya A, et al. Resistance profile for pathogens causing urinary tract infection in a pediatric population, and antibiotic treatment response at a University Hospital, 2010-2011. Colomb Med (Cali). 2014; 45(1): 39-44.

14. Herrera C, Navarro D, Täger M. Etiology and antimicrobial resistance profile of urinary tract infection in children, Valdivia 2012. Rev Chilena Infectol. 2014; 31(6):757-8. doi: 10.4067/S071610182014000600019.

15. Conway PH, Cnaan A, Zaoutis T, Henry BV, Grundmeier RW, Keren R. Recurrent urinary tract infection in children: Risk factors and association with prophylactic antimicrobials. JAMA. 2007; 298 (2): 179-86.

16. Shaikh N, Morone NE, Bost JE, Farrell $\mathrm{MH}$. Prevalence of urinary tract infections in childhood: A Meta-analysis. Pediatr Infect Dis J. 2008; 27(4): 302-08. doi: 10.1097/INF.0b013e31815e4122

17. Muntaha ST, Ismail M, Hassan F. Causative Organisms and their Sensitivity Pattern of UrinaryTract Infection in Children. JIIMC. 2016; 11 (4): 145-48.

18. Hussain M, Ahmad B, Bhatti N, Husain S. Bacteriological Spectrum and Sensitivity Pattern in Culture Proven Urinary Tract Infection in Children. JRMC. 2017; 21(3): 290-92.

19. Haq I, Lal A, Ullah A, Ahmad A, Akram A, Siraj S. Frequency of urinary tract infection in children and their sensitivity pattern in swat JSMC. 2017; 7(1): 8-11.

20. Amir S, Rahim F, Afridi JM. Urinary Tract Infections in Children. J Med Sci. 2013; 21(1): 13-15.

21. Shah S, Ahmad A, Gul I, Rehman G. Etiology and Antibiotic resistance patern of Community acquired urinary tract infection in children. KJMS. 2015; 8(3): 428-33.

22. Gul Z, Jan AZ, Liaqat F, Qureshi MS. Causative organisms and antimicrobial sen-sitivity pattern of pediatric urinary tract infections. Gomal J Med Sci. 2015; 13:118-2.

23. Mobeen A. Microorganisms and Drug Sensitivity Patterns in Urinary Tract Infections. PJMHS. 2013; 7(2): 281-7.

AUTHOR AFFILIATION:

\section{Dr. Deve Dass}

Assistant Professor, Department of Pediatrics

Liaquat University of Medical \& Health Sciences

(LUMHS), Jamshoro, Sindh-Pakistan.

Dr. Muhammad Nadeem Chohan (Corresponding Author)

Assistant Professor, Department of Pediatrics

LUMHS, Jamshoro, Sindh-Pakistan.

Email: nadeem.chohan@lumhs.edu.pk

\section{Dr. Srichand Talreja}

Senior Registrar, Department of Pediatric

Hamdard College of Medicine and Dentistry

Karachi, Sindh-Pakistan. 